Presented at: $7^{\text {th }}$ Advanced Accelerator Concepts Workshop

Lake Tahoe, CA

October 12-18, 1996

BNL- 63684

\title{
Prospects for Compact High-Intensity \\ Laser Synchrotron X-ray and Gamma Sources
}

I.V. Pogorelsky

$$
\text { CONF-9610210--9 }
$$

Accelerator Test Facility, Brookhaven National Laboratory, NSLS, Upton, NY 11973

\section{RECENED \\ JAN 021997 \\ OSTI}

November 1996

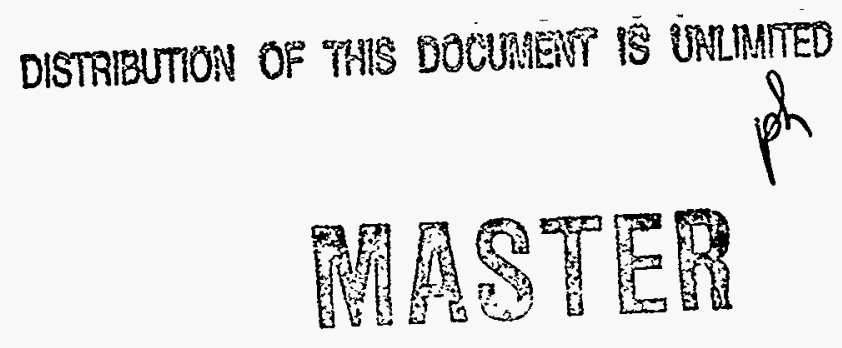

National Synchrotron Light Source

Brookhaven National Laboratory

Upton, NY 11973

Work performed under the auspices of the U.S. Department of Energy, under contract DE-AC02-76CH00016 


\section{DISCLAMER}

Portions of this document may be illegible in electronic image products. Images are produced from the best available original document. 


\section{DISCLAIMER}

This report was prepared as an account of work sponsored by an agency of the United States Government. Neither the United States Government nor any agency thereof, nor any of their employees, make any warranty, express or implied, or assumes any legal liability or responsibility for the accuracy, completeness, or usefulness of any information, apparatus, product, or process disclosed, or represents that its use would not infringe privately owned rights. Reference herein to any specific commercial product, process, or service by trade name, trademark, manufacturer, or otherwise does not necessarily constitute or imply its endorsement, recommendation, or favoring by the United States Government or any agency thereof. The views and opinions of authors expressed herein do not necessarily state or reflect those of the United States Government or any agency thereof. 


\title{
PROSPECTS FOR COMPACT HIGH-INTENSITY LASER SYNCHROTRON X-RAY AND GAMMA SOURCES
}

\author{
I.V. Pogorelsky \\ Accelerator Test Facility, Brookhaven National Laboratory, 725C, Upton, NY 11973
}

\begin{abstract}
$\underline{\text { Abstract }}$
A laser interacting with a relativistic electron beam behaves like a virtual wiggler of an extremely short period equal to half of the laser wavelength. This approach opens a route to relatively compact, high-brightness $\mathrm{x}$-ray sources altemative or complementary to conventional synchrotron light sources. Although not new, the laser synchrotron source (LSS) concept is still waiting for a convincing demonstration. Available at the BNL Accelerator Test Facility (ATF), a high-brightness electron beam and the high-power $\mathrm{CO}_{2}$ laser may be used as prototype LSS brick stones. In a feasible demonstration experiment, 10-GW, 100-ps $\mathrm{CO}_{2}$ laser beam will be brought to a head-on collision with a 10-ps, $0.5-\mathrm{nC}, 50$ $\mathrm{MeV}$ electron bunch. Flashes of collimated $4.7 \mathrm{keV}(2.6 \AA)$ x-rays of 10-ps pulse duration, with a flux of $\sim 10^{19}$ photons/sec, will be produced via linear Compton backscattering. The $x$-ray spectrum is tunable proportionally to the ebeam energy. A rational short-term extension of the proposed experiment would be further enhancement of the $x$-ray flux to the $10^{22}$ photons/sec level, after the ongoing ATF $\mathrm{CO}_{2}$ laser upgrade to $5 \mathrm{TW}$ peak power and electron bunch shortening to $3 \mathrm{ps}$ is realized.

In the future, exploiting the promising approach of a high-gradient laser wake field accelerator, a compact "tabletop" LSS of monochromatic gamma radiation may become feasible.
\end{abstract}

\section{INTRODUCTION}

Generation and application of high-brightness, quasi-monochromatic $x$-rays and $\gamma$-rays is a fast developing area of science and technology. Contemporary synchrotrons equipped with wiggler magnets are the sources of the most intense $x$-ray fluxes, approaching the level of $10^{18}$ photon/sec within the spectral window of $0.1 \%$ of the photon energy. Diverse demands of multidisciplinary research, industrial, and medical applications drive the search for even more intense (as well as compact) $x$-ray sources. Meeting these requirements, the next generation synchrotron light sources (SLS) are under development based on the principles of the free-electron laser.

Another approach to a relatively compact $x$-ray LSS is based on using contemporary picosecond lasers of ultrahigh peak power. The laser beam acts on relativistic electrons as an electromagnetic wiggler but has a period 104-105 times shorter than the conventional undulator. Thus, LSS permits generation of proportionally heavier photons at the same e-beam energy as a conventional SLS. Similarly, LSS permits the use of $\sim 100$ times less energetic electrons to generate $\mathrm{x}$-rays of a particular wavelength, significantly downsizing the required electron accelerator.

Limited by the repetition rate of the laser driver, LSS does not compete with conventional synchrotron sources in average radiation power. However, it has the potential of delivering extremely intense $\mathrm{x}$-ray flashes within picosecond and femtosecond time intervals. X-ray beams with $10^{10}-10^{11}$ photons/pulse may be produced via relativistic Compton scattering (which is a quantum-mechanical foundation of the LSS) using state-of-the-art terawatt picosecond lasers and high-brightness $\mathrm{RF}$ linacs that operate with picosecond photocathode injectors. Such pulses are of particular interest for a number of applications.

Why have these attractive features of the LSS not been utilized so far? Maybe because the appropriate laser and linac have yet to shake hands within one facility. We believe that at the ATF[1] we have such a unique opportunity. Indeed, the ATF's linac, the world's-brightest, delivers up to $0.5 \mathrm{nC}, 10-0.4 \mathrm{ps}, 50-\mathrm{MeV}$ electron bunches with a peak corrent of $\sim 100 \mathrm{~A}$, momentum spread of $0.2 \%$, and normalized emittance down to $0.5 \mathrm{~mm}$.mrad. [2] That permits the high concentration of relativistic electrons necessary for efficient photon scattering. In addition, the ATF-operated picosecond $\mathrm{CO}_{2}$ laser, which delivers $\sim 10$ times more photons than more common solid state picosecond lasers of a similar energy, permits the proportional increase of $x$-ray flux. 
In Section II, we discuss the efficiency of $\mathrm{x}$-ray generation via Compton backscattering of the $\mathrm{CO}_{2}$ laser photons on a counter-propagating relativistic electron beam. As an example, in Section III we consider a feasible demonstration experiment at the ATF where the 10-GW pulses of 100-ps duration (from the presently operational $\mathrm{CO}_{2}$ laser) will be brought into a head-on collision with the $0.5 \mathrm{nC}, 10$-ps bunches of the $50-\mathrm{MeV}$ electron beam. The $4.7 \mathrm{keV}(2.6 \AA) \mathrm{x}$-rays of 10-ps pulse duration with a peak flux of $2 \times 1019$ photons/s will be obtained within the $0.4 \%$ bandwidth window. At the second stage of the ATF experiment, after the ongoing laser upgrade to the $\sim 5 \mathrm{TW}$ power level and the electron bunch shortening to $3 \mathrm{ps,}$ x-ray flashes with peak flux of up to $2 \times 10^{22}$ photons $/ \mathrm{sec}$ may be produced.

As the next step to even more compact and economical $x$-ray and gamma sources, we consider substitution of a conventional electron accelerator with the projected ultra-high gradient laser accelerator. The enthusiasm that drives research in the area of laser acceleration is based on ultra-high electric fields, up to $10^{11} \mathrm{~V} / \mathrm{cm}$, attainable upon the tight focusing of terawatt laser beams. Being used for particle acceleration, such fields may permit reduction of accelerator dimensions by many orders of magnitude, putting SLS on a table top. After demonstration of a $\sim 100 \mathrm{GeV} / \mathrm{m}$ acceleration gradient over a few millimeters of interaction region in the recent laser wakefield accelerator (LWFA) experiment[3], the next goal for the laser accelerator development is to extend the interaction region over a longer distance and to ensure the production of a good quality electron beam. The primary approach to longer interaction distances is based on laser waveguiding in dielectric or plasma channels. In Section IV, we consider the possibility of using the channeled LWFA to produce an electron beam with parameters required for a high-brightness LSS operating in the gamma-region and discuss considerations for choosing a particular type of laser to drive such a facility.

So far, the prime attention of researchers in the area of strong-field laser applications was attracted to the fast advancing technology of table-top terawatt $\left(\mathrm{T}^{3}\right)$ solid state lasers that operate at wavelengths of $\lambda \approx 1 \mu \mathrm{m}$. A picosecond terawatt $\mathrm{CO}_{2}\left(\mathrm{psTW}-\mathrm{CO}_{2}\right)$ laser may be suggested as another candidate for the LSS driver. The first laser of this kind is under development at the ATF.[4]. In the present paper, we show how the LWFA-LSS concept may benefit from the relatively long wavelength $(\lambda \approx 10 \mu \mathrm{m}) \mathrm{CO}_{2}$ laser driver. Up to $250 \mathrm{MeV}$ electron acceleration over a $4 \mathrm{~cm}$ interaction region with a plasma-channeled $5 \mathrm{TW} \mathrm{CO}_{2}$ laser beam is predicted.[5] A feasible high-intensity LSS is based on using split beams of the psTW- $\mathrm{CO}_{2}$ laser to accelerate copropagating electrons and, then, to generate up to $5 \mathrm{MeV}$ gamma-rays at a peak flux above $10^{23}$ photons/sec in head-on collision with electrons within the same compact interaction chamber.

\section{LINEAR COMPTON SCATTERING OF $\mathrm{CO}_{2}$ LASER RADIATION FROM A RELATIVISTIC ELECTRON BEAM}

The advantage of using a laser as a virtual wiggler in the $\mathrm{x}$-ray LSS stems from the equation for the wavelength of photons backscattered from a relativistic electron beam,

$$
\lambda_{\mathrm{x}}=\lambda / 4 \gamma^{2} \text {, }
$$

where $\gamma$ is the relativistic Lorentz factor. Short laser wavelength, $\lambda$, which is normally in the range of $0.3-10 \mu \mathrm{m}$, permits relatively easy access to the $x$-ray and gamma-ray region.

A comprehensive theoretical analysis of Compton photon scattering from relativistic electron beams may be found elsewhere[6,7]. Here, we compile and derive the expressions that help us to understand how the efficiency of $\mathrm{x}$-ray Compton scattering depends upon the laser wavelength and other laser and e-beam parameters.

An electron, oscillating in an external electromagnetic (EM) field, emits radiation at a power of

$$
P_{e}=2 e^{2} \dot{V}^{2} / 3 c^{2}
$$

where $\dot{V}$ is the acceleration vector (averaged over the oscillation period) defined by the acting oscillatory electric field, $\left|E_{L}\right|$, of the EM wave,

$$
\dot{V}=\hat{A}\left|E_{L}\right| / 1
$$

and $\left|E_{L}\right|$ is related to the laser intensity af $P_{\mathrm{A}} / S_{\mathrm{A}}$ via

$$
\left|E_{L}\right|^{2}=2 \mathrm{a}_{\mathrm{A}} / \varepsilon_{\mathrm{A}} \text {. }
$$

Here, $P_{\mathrm{A}}$ is the laser beam power, $S_{\mathrm{A}} \mathrm{AA}{ }_{\mathrm{A}}^{\mathrm{A}}$ is the effective cross-section for the laser beam, and $r_{\mathrm{A}}$ is the laser beam radius. Combining Eqs.(2) through (4), we obtain: 


$$
P_{e}=\frac{4 c}{3 \pi \varepsilon_{0}} P_{L}\left(\frac{r_{e}}{r_{L}}\right)^{2},
$$

where $r_{e}=e 2 / m c^{2}=2.82 \times 10^{-13} \mathrm{~cm}$ is the classical electron radius.

The expression for $P_{e}$, corrected for the relativistic electron motion in a Gaussian laser beam, is [6]

$$
P_{e}=\frac{64}{3} P_{L} \gamma^{2}\left(\frac{r_{e}}{r_{L}}\right)^{2} \text {. }
$$

Considering for simplicity a rectangular laser pulse of duration $\tau_{L}$ and a uniform electron distribution with a density $n_{e}$ over the e-bunch duration $\tau_{b}$, the power radiated by the electron ensemble within the immediate interaction region $L(t)$ is equal to

$$
P_{X}(t)=P_{e} n_{e} L(t) S_{L}
$$

where $L(t)$ at any moment $t$ is equal to or smaller than $\min \left\{c \tau_{L}, c \tau_{b}\right\}$. Here, we consider counter-propagating electron and laser beams because this geometry ensures the maximum number of generated $x$-ray photons for given laser and $e$ beam parameters. The radiation process will continue during the time interval while the laser pulse and the electron bunch are at least in a partial overlap. It is presumed that the e-beam radius $r_{b}$ matches, or is bigger than, $r_{L}$. That ensures that the total laser power is used efficiently. Then, the total backward scattered radiation energy is

$$
\mathrm{E}_{X}=\int_{0}^{\left(\tau_{L}+\tau_{b}\right) / 2} P_{X}(t) \partial t=P_{e} n_{e} S_{L} c\left[\min \left\{\tau_{L}, \tau_{b}\right\}\left(\frac{1}{2} \min \left\{\tau_{L}, \tau_{b}\right\}+\mid \frac{\tau_{L}-\tau_{b}}{2}\right)\right]=P_{e} n_{e} S_{L} c \frac{\tau_{L} \tau_{b}}{2} .
$$

Note that the actual duration of the produced backscattered pulse is not equal to the total laser-electron interaction time interval, $\tau_{\text {int }}=\left(\tau_{L}+\tau_{b}\right) / 2$. Since $x$-rays originate from electrons in the direction of the electron propagation, it turns out that the $x$-ray pulse length is defined primarily by the electron bunch duration. On top of it is the $\mathrm{x}$-ray pulse stretching due to the $\mathrm{x}$-ray and electron speed mismatch accumulated over the interaction time,

$$
\Delta \tau=(1-\beta) \frac{\tau_{L}+\tau_{b}}{2} \approx \frac{\tau_{L}+\tau_{b}}{4 \gamma^{2}}
$$

Hence, the total $\mathrm{x}$-ray pulse duration is

$$
\tau_{X}=\tau_{b}+\Delta \tau=\frac{1-\beta}{2} \tau_{L}+\frac{3-\beta}{2} \tau_{b} \approx \tau_{b}+\frac{\tau_{L}}{4 \gamma^{2}} .
$$

However, for the practically important case when $\tau_{L} \ll 4 \gamma^{2} \tau_{b}$, we can consider $\tau_{X} \approx \tau_{b}$. It follows that relatively long laser pulses may be tolerated without a noticeable increase in the $x$-ray pulse duration above $\tau_{b}$. Combining Eqs.(6), (8) and (10), the expression for $x$-ray power is

$$
P_{X}=\frac{\mathrm{E}_{X}}{\tau_{X}} \approx \frac{32 \pi}{3} \mathrm{E}_{L} n_{e} c \gamma^{2} r_{e}^{2} \approx \frac{10 \mathrm{E}_{L} Q \gamma^{2} r_{e}^{2}}{e \tau_{b} r_{b}^{2}},
$$

where $E_{L}=P_{L} \tau_{L}$ is the laser pulse energy, $Q$ is the electron bunch charge and $r_{b}$ is the e-beam radius in the interaction region (we consider $\left.r_{b}\right\rangle_{\mathrm{L}}$, so that no laser energy is lost for interaction). Corresponding to Eq.(11), the engineering formula is

$$
P_{X}[W] \approx \frac{0.5 \mathrm{E}_{L}[J] Q[\mathrm{nC}] \gamma^{2}}{\tau_{b}[\mathrm{ps}] \mathrm{r}_{b}^{2}[\mathrm{~mm}]}
$$

The conclusion drawn from Eq.(11) is that the intensity of the produced $x$-rays does not depend directly upon the laser pulse duration. The high total laser pulse energy, short-duration of the relativistic electron bunch, and high density of the electrons within the bunch become the most important parameters that define the $x$-ray peak power. In addition, in order to be in a good overlap, the laser beam shall be focused to the same or smaller spot size as the electron beam.

Because of the natural divergence of the electron and laser beams, they remain tightly focused only within a limited distance. For the Gaussian optical beam, this distance is related to the Rayleigh length, $z_{0}$, measured from the focal point to the point where the laser beam expands two times in a cross-sectional area,

$$
L_{L} \approx \pi z_{0}=\pi^{2} r_{L}^{2} / \lambda \text {. }
$$


A similar condition is applicable to electron beams with their divergence characterized by geometric emittance $\varepsilon / \gamma$ in the same way as diffraction-limited laser beams are characterized by wavelength,

$$
L_{b}=\pi^{2} r_{b}^{2} \gamma / \varepsilon \text {. }
$$

The comparison of $\lambda$ with $\varepsilon / \gamma$ tells which one, e-beam or laser beam, is the limiting factor for making the interaction region narrow. Typical low-emittance electron beams provided by RF linacs permit much tighter filamentation than focused laser beams. Thus, we consider that limitations to the interaction length are imposed by the laser beam.

For the efficient use of the laser pulse in the Compton process, the laser beam waist length, $L_{L}$, shall extend over the overlap distance defined by the electron and laser pulse duration,

$$
L_{L} \geq c\left(\tau_{L}+\tau_{b}\right) / 2 \text {. }
$$

If the interaction distance is below the limit defined by Eq.(15), it may result in the proportional drop of the laser energy coupled to the electron bunch. Thus, overly-tight laser focusing would not help to gain any additional x-ray power. From Eqs.(13) and (15), it follows that, at $\tau_{L} \geq \tau_{b}$, laser parameters shall also satisfy the condition

$$
r_{L}^{2} \geq \frac{c \tau_{L} \lambda}{2 \pi^{2}}
$$

Combining Eqs.(12) and (16), we obtain the estimate for the maximum attainable $\mathrm{x}$-ray power, $P_{X}^{\max }$, when the laser beam divergence is the limiting factor for the length and cross-section of the interaction area,

$$
P_{X}^{\max }[k W] \approx \frac{30 P_{L}[T W] Q[n C] \gamma^{2}}{\tau_{b}[p s] \lambda[\mu m]} .
$$

Note, that Eq.(12) is still valid when realistic $r_{b}$ is above the limit set by Eq.(16).

Power of the $\mathrm{x}$-ray beam is just one of parameters entering into the expression for spectral brightness.

$$
B=\frac{P_{X}}{\left(2 \pi R \theta_{\Delta v}\right)^{2} \Delta v_{X}} \text {. }
$$

Here, $\theta_{\Delta v}$ is the angular divergence of the radiation within the spectral bandwidth $\Delta v_{X}$. The total angular divergence of the backscattered radiation is defined by $\gamma$,

$$
\theta_{0}=1 / \gamma \text {. }
$$

Within this cone, the scattered radiation frequency drops off axis according to

$$
\Delta v_{X} / v_{X} \approx \gamma^{2} \Delta \theta^{2}
$$

thus, $\Delta v_{X} / v_{X} \approx 1$ over $\Delta \theta=\theta_{0}$.

It follows from Eq.(1) that the bandwidth of the backscattered $x$-rays is directly related to the momentum spread or the "temperature" of the e-beam as

$$
\Delta v_{X} / v_{X}=2 \Delta \gamma / \gamma \text {. }
$$

This narrow bandwidth radiation is observed within the cone with the opening angle

$$
\theta_{\Delta v} \approx\left(\Delta v_{X} / v_{X}\right)^{1 / 2} / \gamma \text {. }
$$

From the above expressions for $P_{X}, \Delta v_{X}$, and $\theta_{\Delta v}$ we can conclude that, in order to produce high-brightness $\mathrm{x}$ rays for a particular wavelength, it is beneficial to choose possibly high values for both parameters, $\gamma$ and $\lambda$. To illustrate this conclusion, let us compare $\mathrm{CO}_{2}(\lambda=10 \mu \mathrm{m})$ and Nd:YAG $(\lambda=1 \mu \mathrm{m})$ lasers of equal power. As long as $\lambda_{X}=\lambda / 4 \gamma^{2}$ is considered as an invariant, then choosing the $\mathrm{CO}_{2}$ laser, with its wavelength 10 times longer than the solid state laser wavelength, requires a $\sqrt{10}$ more energetic e-beam. These leads to the improvement of the angular divergence of the produced X-rays which is equal to $1 / \gamma$, and reduction of spectral bandwidth defined by $\Delta \gamma / \gamma$. According to $P_{X} \sim E_{L} \gamma^{2}$ (see Eq.(12)), the backscattered $x$-ray power will rise 10 times due to the higher $\gamma$. This actually stems from the facts that the $\mathrm{x}$-ray flux is proportional to the delivered laser photon flux, and, at the fixed laser energy, the last parameter is proportional to $\lambda$. When combining together all of the mentioned above, we come to the conclusion that using a $\mathrm{CO}_{2}$ laser as the LSS driver opens the prospect for up to 300 times increase in the spectral brightness of the produced $x$-rays of a particular wavelength compared with using a $1-\mu \mathrm{m}$ laser of the same energy.

The above remark on the advantage of using a long-wavelength $\mathrm{CO}_{2}$ laser is valid provided other parameters entering to Eq.(12) are equal. In a situation when the cross-section of the interaction region is limited by the diffraction 
divergence of the laser beam, which is proportional to $\lambda, P_{X}$ does not depend any more upon $\lambda$. This conclusion follows also from Eq.(17) under the assumed above condition of $\gamma \sim \sqrt{\lambda}$. However, it is most likely that the radius of the interaction region will not be reduced much below $30 \mu \mathrm{m}$ due to such practical considerations as alignment and pointing stability of the laser and e-beam. Focusing to $r_{L}=30 \mu \mathrm{m}$ may be obtained with the $\mathrm{CO}_{2}$ laser as well. Under this condition, the wavelength power scaling discussed above is still valid.

\section{DESIGN PARAMETERS FOR THE ATF LSS EXPERIMENT}

LSS, based on linear relativistic Compton backscattering of picosecond $\mathrm{CO}_{2}$ laser pulses, may be realized at the ATF with its presently available $10 \mathrm{GW}, 100$-ps $\mathrm{CO}_{2}$ laser and 10-ps, $0.5 \mathrm{nC}, 50 \mathrm{MeV}(\gamma=100)$ electron beam. For the electron beam, we assume the normalized emittance of $\varepsilon=2 \mathrm{~mm} \cdot \mathrm{mrad}$ and momentum spread of $\delta p / p=0.2 \%$, as already demonstrated at the ATF. Quantitative estimates for the parameters of the feasible ATF LSS experiment are done in Tablel (Stage 1).

By Eq.(1), the wavelength of the backscattered radiation will be $\lambda_{x}=\lambda / 4 \gamma^{2}=2.6 \AA$. That corresponds to the photon energy of $h v_{X}[\mathrm{eV}]=1.25 \times 10^{4} / \lambda_{X}[\AA]=4.7 \mathrm{keV}$.

Spectral tuning of the generated $x$-rays is possible via $\gamma$ adjustment. The $x$-ray power, Eq.(12), will vary proportionally to $\gamma^{2}$, and the $\mathrm{x}$-ray brightness, Eq.(18), proportionally to $\gamma^{5}$. Another way for fine tuning the $\mathrm{x}$-ray wavelength is by tuning the $\mathrm{CO}_{2}$ laser wavelength within the $\mathrm{CO}_{2}$ gain spectrum $(9.2-11.8 \mu \mathrm{m})$.

According to Eq.(15), the length of the interaction region, where the focused laser beam shall match in a crosssection the counter-propagating e-beam, is $L_{i n}=17 \mathrm{~mm}$.

As discussed above, the comparison of $\lambda$ with $\varepsilon / \gamma$ tells which one, e-beam or laser beam, is the principal limiting factor for making the interaction region narrow. The demonstrated at the ATF geometric emittance, $\varepsilon / \gamma=2 \times 10^{-2} \mu \mathrm{m} . \mathrm{rad}$, being orders of magnitude smaller than the $\mathrm{CO}_{2}$ laser wavelength, $\lambda=10.6 \mu \mathrm{m}$, indicates that the $\mathrm{CO}_{2}$ beam diffraction divergence will ultimately define the effective radius of the interaction region. Using Eq.(13), we calculate the waist radius of the diffraction limited $\mathrm{CO}_{2}$ laser beam to be $\eta_{2}=\sqrt{L_{i n t} \lambda} / \pi \approx 140 \mu \mathrm{m}$. Hence, the e-beam shall be focused to the same size, $r_{b}=140 \mu \mathrm{m}$

At the specified laser and e-beam parameters, the $\mathrm{x}$-ray flux is $2 \times 1019$ photons/sec and the total number of the $\mathrm{x}$ ray photons per pulse is equal to $2 \times 10^{8}$ photons/pulse.

Utilizing prospective features of the ATF that are under development, we may project an advancement to the Stage 2 of the ATF LSS experiment. For the upgraded LSS version, consider a $70 \mathrm{MeV}$, 3-ps electron bunch, attained via compression of the photocathode UV laser driver pulse in a nonlinear crystal[8], and a 5-TW 3-ps $\mathrm{CO}_{2}$ laser.[4]

Note, that with a short, 3-ps laser pulse, the x-ray intensity increase will be attained, according to Eq.(12), via shortening of the interaction region that permits tighter focusing of the laser and electron beams. With the short interaction length $L_{i n}=1 \mathrm{~mm}$ (defined by the short laser and electron pulses, both $3 \mathrm{ps}$ ), the laser beam waist may be brought to $r_{L}=30 \mu \mathrm{m}$. Assuming the corresponding focusing of the e-beam, the $9.36 \mathrm{keV} \mathrm{x}$-ray peak flux will be increased to $2 \times 10^{22}$ photons/sec. The total $x$-ray photon number in this case is $6 \times 10^{10}$ photons/pulse. At the short, 3-ps laser pulse, the $x$-ray spectrum is defined not just by the e-beam temperature, as according to Eq.(21), but will broaden to

$$
\Delta \lambda^{\prime} \times / \lambda_{\mathrm{x}}=1 / N \text {, }
$$

where $N$ is the total number of laser wavelengths during the electron-laser interaction, that is 100 for $\tau_{L}=3 \mathrm{ps,} \mathrm{thus,}$

$\Delta \lambda_{x}{ }^{\prime} / \lambda_{x} \approx 0.6 \%$. The angle-averaged brightness is $B(0.6 \%$ b.w. $) \approx 2 \times 10^{21}$ photons $/ \mathrm{sec}^{2} \mathrm{~mm}^{2} \mathrm{mrad}^{2}$ or $\mathrm{B}(0.1 \%$ b.w. $) \approx 3 \times 10^{20}$ photons/sec. $\mathrm{mm}^{2} \mathrm{mrad}^{2}$.

It becomes evident that, in order to maintain high $\mathrm{x}$-ray monochromaticity, the $\mathrm{CO}_{2}$ laser pulse duration shall be relatively long, $\tau_{L} \geq 30 \mathrm{ps}$. The contradiction involved in the desire to have a long interaction distance with a narrow crosssection may be resolved by channeling the laser beam. The idea of laser beam channeling, relevant also for $x$-ray lasers, laser acceleration in plasma, and laser fusion, is presently under extensive study. Two primary approaches look feasible and have been demonstrated experimentally: dielectric waveguide[9] and preformed plasma channel[10].

With a $5-\mathrm{TW} \mathrm{CO}_{2}$ laser beam focused to a $30 \mu \mathrm{m}$ diameter spot, as high as $10^{17} \mathrm{~W} / \mathrm{cm}^{2}$ intensity will be attained. Such intensity corresponds to the unitless laser strength of $a=3$ defined by the expression

$$
a=0.85 \times 10^{-9} \lambda[\mu \mathrm{m}] I^{1 / 2}\left[\mathrm{~W} / \mathrm{cm}^{2}\right]=0.3 E_{L}[T V / m] \lambda[\mu \mathrm{m}] .
$$


It is known that at $a>1$, the nonlinear Compton scattering effect comes to the scene[6]. With the maximum order of generated harmonics equal to $n=a^{3}$, as short as $\lambda=0.05 \AA$ radiation may be produced when a $\mathrm{CO}_{2}$ laser beam is backscattered by the $70-\mathrm{MeV}$ e-beam. The maximum of the intensity distribution shifts to $n=11-13$, with these components $\sim 3$ times more intense than the fundamental $n=1$ component.[6]

Other interesting features of harmonics are that their angular divergence and spectral bandwidth are inversely proportional to $n$, offering prospects for considerable increase in a spectral brightness. Study of nonlinear Compton scattering may be carried out as the next stage of the ATF LSLS experiment.

The parameters of the advanced LSS summarized in Table 1 (Stage 2) are calculated for linear Compton scattering only. In addition, a multitude of high-brightness harmonics will be observed as discussed above.

Table 1. Design Parameters of the ATF X-Ray LSS Experiment

\begin{tabular}{|c|c|c|}
\hline Experiment Staging: & Stage 1 & Stage 2 \\
\hline \multicolumn{3}{|l|}{ ELECTRON BEAM } \\
\hline Energy $[\mathrm{MeV}]$ & 50 & 70 \\
\hline Bunch Charge [nC] & 0.5 & 0.5 \\
\hline Bunch Duration FWHM [ps] & 10 & 3 \\
\hline Radius at Focus $[\mu \mathrm{m}]$ & 140 & 30 \\
\hline Waist Length [mm] & $>100$ & $>10$ \\
\hline Normalized Emittance [mm.mrad] & 2 & 2 \\
\hline Electron Momentum Spread [\%] & 0.2 & -0.05 \\
\hline \multicolumn{3}{|l|}{$\mathrm{CO}_{2}$ LASER } \\
\hline Pulse Duration [ps] & 100 & 3 \\
\hline Peak Power [GW] & 10 & 5000 \\
\hline Laser Energy [J] & 1 & 15 \\
\hline Radius at Focus [ [ $\mathrm{m}]$ & 140 & 30 \\
\hline Waist Length [mm] & 17 & 1 \\
\hline \multicolumn{3}{|l|}{ XRAYS } \\
\hline Wavelength $[\AA]$ & 2.6 & 1.35 \\
\hline Pulse Duration [ps] & 10 & 3 \\
\hline Angular Spread [mrad] & 10 & 7 \\
\hline Spectral Bandwidth [\%] & 0.4 & 0.6 \\
\hline Photons per Pulse & $2 \times 10^{8}$ & $6 \times 10^{10}$ \\
\hline Peak Flux [photons/s] & $2 \times 10^{19}$ & $2 \times 10^{22}$ \\
\hline Peak Brightness (/0.1\%) [photon/sec. $\left.\mathrm{mm}^{2} \mathrm{mrad}^{2}\right]$ & $10^{16}$ & $3 \times 10^{20}$ \\
\hline
\end{tabular}

\section{TABLE-TOP LASER WAKEFIELD $\gamma$-RAY SYNCHROTRON SOURCE}

The idea of a laser wakefield electron accelerator (LWFA)[11,12], that is currently under intense study, leads to record accelerating gradients, hundreds times higher than with conventional linacs.[3] A combination of plasma accelerator with LSS may open a route to the extremely compact, table-top wakefield $x$-ray and gamma LSS.

Another feature important for the LSS is the high quality of the produced e-beam that includes: a small (close to $0.1 \%$ ) energy spread, and a low emittance. In order to generate a high-quality e-beam in the LWFA, we might want to start with a similar or better quality seed e-beam and preserve it in the course of the laser acceleration in plasma. The requirement for small divergence of the Compton backscattered photons puts a limit on the e-beam normalized emittance: $\varepsilon[\mathrm{mm} . \mathrm{mrad}]<<r_{L}[\mu \mathrm{m}]$. The requirement for the small energy spread presumes that the seed electron bunches should be much shorter than the plasma wake period 


$$
\tau_{b} \ll<\lambda_{p} / c,
$$

where plasma wavelength, $\lambda_{p}$, is related to the plasma density $N_{e}$ via

$$
N_{e}=10^{19}\left(\frac{\lambda}{\lambda_{p}}\right)^{2}\left[\mathrm{~cm}^{-3}\right] \text {. }
$$

The $0.1 \%$ energy spread calls for a monochromatic electron bunch of the $\tau_{b} \leq \lambda_{p} / 30 c$ duration. It follows from Eq.(26) that the long wavelength of the $\mathrm{CO}_{2}$ laser permits a 100 times plasma density reduction in comparison with the more common solid state lasers that have $\lambda \approx 1 \mu \mathrm{m}$. The reduced electron scattering in a low density plasma will help to preserve a low e-beam emittance.

Contemporary photocathode RF guns may serve as injectors of electrons with the required parameters. In particular, $370 \mathrm{fs}$ electron bunches of $5 \mathrm{MeV}$ energy, $40 \mathrm{pC}$ charge $\left(2.5 \times 10^{8}\right.$ electrons), $0.15 \%$ energy spread, and 0.5 mm.mrad normalized emittance have been demonstrated at the ATF by proper phasing of photoelectrons with RF field.[2] As short as $100 \mathrm{fs}$ bunches may be expected after further development of this technique.

Let us consider a prospective LWFA driven by a 5 TW $\mathrm{CO}_{2}$ laser. For the standard LWFA scheme[11], the duration of the driver laser pulse shall satisfy the requirement

$$
\tau_{L} \approx \lambda_{p} / 2 c \text {. }
$$

It is understood that, due to the natural diffraction of the laser beam, the interaction length may be limited to a few millimeters. Thus, some sort of laser beam channeling is required. The condition for plasma waveguiding is[13]

$$
\Delta N_{e}\left[\mathrm{~cm}^{-3}\right]=1.1 \times 10^{20} / r_{L}^{2}[\mu \mathrm{m}] \text {. }
$$

Here, $\Delta N_{e}$ is the height of a wall in a plasma channel that is required to guide a laser beam.

Thus, we see that $\tau_{b}$ and $\tau_{L}$ define $\lambda_{p}$ through Eqs.(25) and (27), and $N_{\varepsilon}$ via Eq.(26). At the same time, the chosen plasma density limits the laser focal spot size to the radius $r_{L}$ defined by Eq.(28), thus limiting the attained laser field, $E_{L}$, to the value

$$
E_{L}[T V / m]=2.6 \times 10^{-9} I_{L}^{1 / 2}\left[W / \mathrm{cm}^{2}\right]=15 \times P_{L}^{1 / 2}[T W] r_{L}[\mu m] .
$$

$N_{c}$ is also one of the factors that defines the efficiency of conversion of the laser EM field into the accelerating wakefield, $E_{a}$,

$$
\eta \equiv E_{a} / E_{L}=1.6 \times 10^{-11} N_{e}^{1 / 2}\left[\mathrm{~cm}^{-3}\right] \lambda[\mu m] a_{L} /\left(1+a_{L}^{2} / 2\right)^{1 / 2} .
$$

Another parameter that enters Eq.(30) is unitless laser strength, $a$, defined by Eq.(24).

This completes the self-consistent set of equations that defines the only solution for plasma and waveguide parameters and, ultimately, the acceleration gradient for the given input laser and e-bunch parameters.

With the theoretically shortest $\mathrm{CO}_{2}$ laser pulse duration of $\tau_{L}=1 \mathrm{ps}$, that has been demonstrated experimentally[14], we obtain wakefield parameters presented in Table 2 (Standard LWFA). With the calculated rather moderate acceleration gradient of $E_{a}=0.5 \mathrm{GV} / \mathrm{m}$, there is still an opportunity to obtain a $50-\mathrm{MeV}$ electron beam after passing a $10 \mathrm{~cm}$ long plasma waveguide.

Conventional linacs are capable to produce up to $100 \mathrm{MV} / \mathrm{m}$ accelerating fields. To demonstrate practical advantages of the new technology for compact LSS in a more pronounced way, the prototype laser accelerator should be capable of producing at least several hundred $\mathrm{MeV}$ electrons in a compact device.

Acceleration gradients much higher than with the standard LWFA, have been demonstrated for, so called, selfmodulated (SM) LWFA regime[12] when laser power, $P_{L}$, satisfies the condition for relativistic self-focusing (RSF):

$$
P_{L} \geq 17\left(\omega / \omega_{p}\right)^{2}[G W]
$$

With a $\mathrm{CO}_{2}$ laser of a $P_{L}=5 \mathrm{TW}$ power, the condition in Eq.(34) is satisfied at $\lambda_{p} \leq 170 \mu \mathrm{m}\left(N_{c} \geq 3.5 \times 10^{16} \mathrm{~cm}^{-3}\right)$. The corresponding wake period is $500 \mathrm{fs}$, which is still much longer than the projected electron bunch duration $(\sim 100 \mathrm{fs})$. Note, that a $1-\mu \mathrm{m}$ laser of similar power requires 10 times smaller $\lambda_{p}$ and, accordingly, 100 times higher plasma density[15]. This will adversely impact both e-beam divergence, due to the multiple scattering in the gas, and energy spread, due to the high proportion of $\tau_{b} c / \lambda_{p}$.

The complexity of the processes involved in the generation of a wake field with a self-modulated relativistically strong laser beam guided in a plasma channel does not allow analytical solution. Simulations performed close to the conditions specified in Table 2 (Self-Modulated LWFA) predict $250 \mathrm{MeV}$ acceleration over a 4-cm interaction 
distance.[5] Even higher, up to $450 \mathrm{MeV}$, acceleration has been simulated for longer interaction distances.[16] It has been shown also that the net acceleration does not depend much upon the initial electron energy, and 5-MeV seed electrons may be used with the same efficiency as $50-\mathrm{MeV}$ electrons. Based on these results, we assume $250 \mathrm{MeV}$ SMLWFA accelerating stage for a table-top LSS in Table 2.

Table 2. Design Parameters for Compact X-Ray and Gamma LSS

\begin{tabular}{|c|c|c|}
\hline Laser Accelerator Type: & $\begin{array}{c}\text { Standard } \\
\text { LWFA }\end{array}$ & $\begin{array}{c}\text { Self-Modulated } \\
\text { LWFA }\end{array}$ \\
\hline \multicolumn{3}{|c|}{ INPUT ELECTRON BEAM } \\
\hline Energy [MeV] & 5 & 5 \\
\hline Bunch Charge [nC] & 0.5 & 0.5 \\
\hline Bunch Duration FWHM [fs] & 100 & 100 \\
\hline Radius at Focus [ $\mu \mathrm{m}]$ & 30 & 30 \\
\hline Waist Length [cm] & 16 & 16 \\
\hline Normalized Emittance [mm.mrad] & 0.5 & 0.5 \\
\hline Electron Momentum Spread [\%] & 0.2 & 0.2 \\
\hline \multicolumn{3}{|c|}{ PLASMA ACCELERATOR } \\
\hline Laser Peak Power [TW] & 5 & 5 \\
\hline Laser Pulse Duration [ps] & 1 & 3 \\
\hline Laser Focus Radius [ $\mu \mathrm{m}]$ & 220 & 60 \\
\hline Laser Waist Length [cm] & 4 & 0.3 \\
\hline Plasma Density $\left[\mathrm{cm}^{-3}\right]$ & $2.3 \times 10^{15}$ & $3.5 \times 10^{16}$ \\
\hline Plasma Wavelength $[\mu \mathrm{m}]$ & 660 & 170 \\
\hline Channel Radius [ $\mu \mathrm{m}]$ & 220 & 60 \\
\hline Channel Length $[\mathrm{cm}]$ & 10 & 4 \\
\hline Acceleration gradient [GV/m] & 0.5 & 6 \\
\hline Energy Gain with Channeling $[\mathrm{MeV}]$ & 50 & 250 \\
\hline \multicolumn{3}{|c|}{ 10-th HARMONIC IN COPMPTON BACKSCATTERING } \\
\hline Laser Peak Power [TW] & 5 & 5 \\
\hline Laser Pulse Duration [ps] & 1 & 3 \\
\hline Laser Focus Radius $[\mu \mathrm{m}]$ & 30 & 30 \\
\hline Laser Waist Length [cm] & 0.1 & 0.1 \\
\hline Photon Energy [keV] & 47 & 4700 \\
\hline Pulse Duration [fs] & 100 & 100 \\
\hline Angular Spread [mrad] & 1 & 0.1 \\
\hline Spectral Bandwidth [\%] & 0.2 & 0.06 \\
\hline Photons per Pulse & $2 \times 10^{10}$ & $6 \times 10^{10}$ \\
\hline Peak Flux [photons/s] & $2 \times 10^{23}$ & $6 \times 10^{23}$ \\
\hline
\end{tabular}

Further simulations are required to determine the quality of the produced e-beam. Preliminary estimates of the electron energy spread are based on the proportion between the e-bunch length and wake period. Normalized emittance of the electron beam is considered not to be effected by the plasma wake.

Linear and nonlinear Compton scattering of laser photons on $250 \mathrm{MeV}$ electrons will produce high-intensity monochromatic hard $\mathrm{x}$-rays and gamma rays in a table-top device that may offer unique capabilities for multidisciplinary research, ultra-high resolution lithography and microscopy.

Parameters in Table 2 are calculated for conditions of laser guiding in a plasma channel. Dielectric capillary tubes may offer another possibility for guiding high-power laser beams in LWFA. This opportunity is based on the finite velocity of the plasma front that is originated at the capillary wall due to the laser ablation. At a typical plasma implosion 
velocity of several $\mathrm{km} / \mathrm{sec}$, it should take several nanoseconds for the overdense plasma to fill a capillary. Thus, the picosecond laser pulse will not be effected by capillary ablation. Tests with a picosecond Nd:YAG laser[9] show the viability of this approach.

The principal schematic of the table-top $\mathrm{x}$-ray LSS is presented in Fig.1. A 5-MeV photocathode electron gun serves as the injector of a high-brightness electron beam. A quadrupole triplet or solenoid focuses the e-beam into the 120 $\mu \mathrm{m}$ wide laser waveguide. The interaction cell is filled with $\mathrm{H}_{2}$ at $\sim 1$ torr and is separated from the gun by the $\leq 1 \mu \mathrm{m}$ thick diamond window. The $\mathrm{CO}_{2}$ laser beam is split into two beams that serve to drive both LWFA and LSS. The delay between the split beams is adjusted optically to make both beams synchronized with the femtosecond electron bunch. The LWFA driver laser beam is focused at the entrance of the waveguide with a parabolic $\mathrm{Cu}$ mirror drilled in the center to transmit the input e-beam. Via tunnel ionization of $\mathrm{H}_{2}$, the laser beam produces plasma of $3.5 \times 10^{16} \mathrm{~cm}^{-3}$ electron density and generates a strong plasma wave. Another parabolic mirror with a central hole serves to focus the LSS driver laser beam at the exit of the waveguide. At this exit, electrons accelerated in the plasma wake field interact with a split portion of the $\mathrm{CO}_{2}$ laser beam generating backscattered $\mathrm{x}$ - or $\gamma$-rays. A downstream dipole serves to separate photons from electrons which are sent to a dump to reduce the $\mathrm{x}$-ray noise. $\mathrm{X}$-rays or gamma quanta are extracted through the output foil window.

Note, that for simplicity of the drawing, we presume a LWFA with a dielectric laser waveguide. In the case of the plasma channel, the accelerator stage will be modified as illustrated in Fig.5 in the reference [15] of these Proceedings. Then, before interacting with the LSS driver laser beam, the accelerated electrons penetrate the hole in the $45^{\circ}$ mirror that serves to direct the third $\mathrm{CO}_{2}$ laser beam to the axicon mirror.

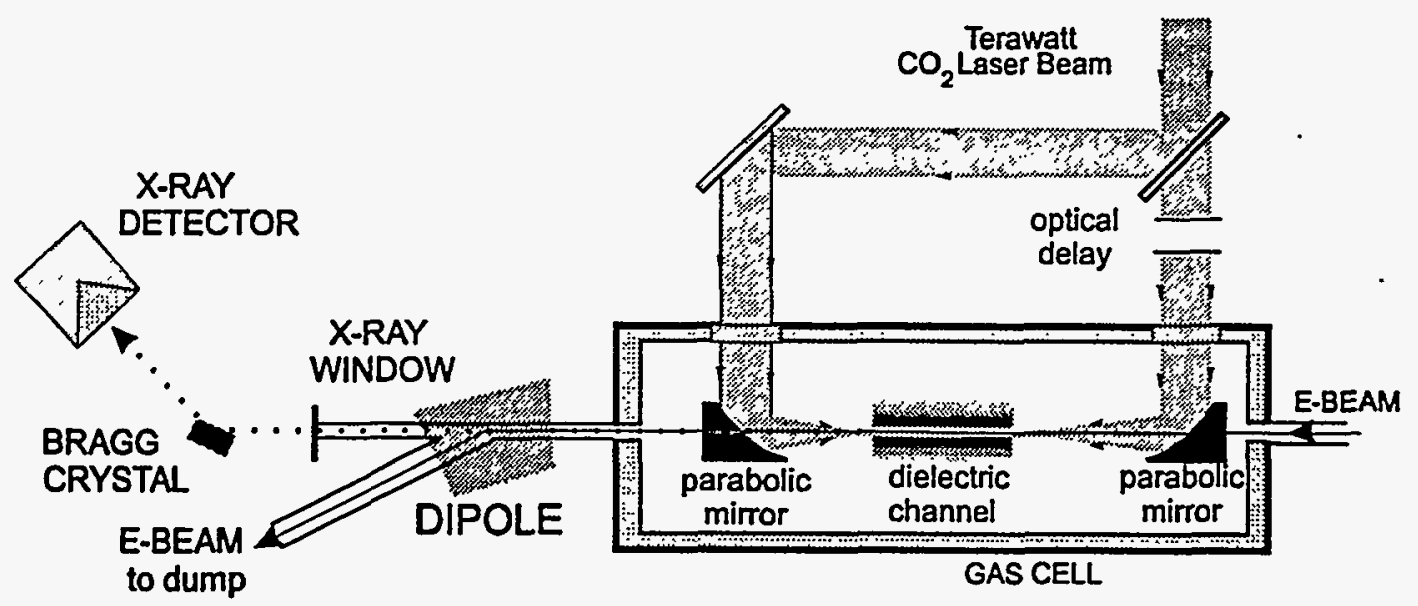

Fig.1 Principal diagram of the compact LSS based on laser wakefield acceleration in a waveguide;

\section{v. CONCLUSIONS}

At the ATF, the world's brightest $50-\mathrm{MeV}$ linac and a high-power picosecond $\mathrm{CO}_{2}$ laser may serve as brick stones for the demonstration Laser Synchrotron Source experiment. After the ongoing laser upgrade to the terawatt power level, monochromatic $x$-ray flashes with peak flux of up to $10^{22}$ photons/sec may be produced, orders of magnitude above the numbers achieved with conventional synchrotron light sources. With a terawatt $\mathrm{CO}_{2}$ laser, the expected nonlinear Compton scattering effect become efficient and may be used for significant expansion of the $\mathrm{x}$-ray spectrum to benefit the potential applications.

As a next step to even more compact and economical x-ray and gamma sources, we consider substitution of a conventional electron accelerator with a high-gradient laser accelerator. We discuss laser wavelength impact on the efficiency of the laser wakefield electron acceleration, quality of the accelerated e-beam, and parameters of the produced gamma radiation, demonstrating advantages of $\mathrm{CO}_{2}$ lasers for such an application. 
$250 \mathrm{MeV}$ electrons and $5 \mathrm{MeV}$ gamma quanta at a flux of $6 \times 10^{23}$ photons/sec may be produced in a table-top device using a conventional photocathode $\mathrm{RF}$ gun and 5-TW $\mathrm{CO}_{2}$ laser that will be commissioned at the ATF in 19971998. The previously discussed feasible LSS may offer unique capabilities for multidisciplinary research, ultra-high resolution lithography, and microscopy.

\section{Acknowledgments}

The author wish to thank I. Ben-Zvi for encouragement of this study and fruitful discussions, T. Srinivasan-Rao, K. McDonald, P. Siddons, J. Hastings, X-J. Wang, J. Skaritka, and K.Kusche for discussions and help in preparation of the upcoming tests.

The work is supported by the US Department of Energy.

\section{$\underline{\text { References }}$}

1. www.bnl.gov/atf

2. X.J. Wang, X. Qui, and I. Ben-Zvi, "Experimental Observation of High-Brightness Micro-Bunching in a Photocathode RF Electron Gun", to be published in Phys. Rev. Letts.

3. A. Madena, Z. Najmudin, A.E. Dangor, et.al., Nature, 377, 606 (1995); T. Katsouleas, "Laser Acceleration of Electrons in Plasmas", Joint Meeting of APS and AAPT, May 2-5, 1996, Indianapolis, IN.

4. I.V. Pogorelsky, I. Ben-Zvi, J. Skaritka, Z. Segalov, M. Babzien, K. Kusche, I.K. Meskovsky, V.A. Lekomtsev, A.A. Dublov, Yu.A. Boloshin, and G.A. Baranov, "The First Terawatt Picosecond $\mathrm{CO}_{2}$ Laser for Advanced Accelerator Study at the Brookhaven ATF", these Proceedings.

5. S.V.Bulanov, T.J. Esirkepov, N.M. Naumova, F. Pegoraro, I.V. Pogorelsky, and A.M. Pukhov, IEEE Trans. on Plasma Sci., 24, 393 (1996)

6. E. Esarey, P. Sprangle, and S.K. Ride, "Nonlinear Thomson Scattering of Intense Laser Pulses from Beams and Plasmas", Preprint NRL/MR/6790-93-7365 (1993)

7. S.K. Ride, E. Esarey, and M. Baine, "Thompson Scattering of Intense Lasers from Electron Beams at Arbitrary Interaction Angles", Phys. Rev. E, 52, \#5 November (1995)

8. A. Umbrasas, J-C. Diels, J. Jacob, G. Valiulis, A. Piskarkas, Opt. Lett., 20, 2228 (1995)

9. S. Jackel, R. Burris, J. Grun, A. Ting, C. Manka, K. Evans, and J. Kosakowskii, Opt. Lett., 20, 1086 (1995)

10. C.G. Durfee III and H.M. Milchberg, Phys. Rev. Lett., 71, 2409 (1993)

11. T. Tajima and J.M. Dawson, Phys. Rev. Lett., 43, 267 (1979)

12. P. Sprangle, E. Esarey, J. Krall, and G. Joice, Phys. Rev. Leii., 69, 2200 (1992)

13. E. Esarey, P. Sprangle, J. Krall, and A. Ting, IEEE Trans. On Plasma Sci., 24, 252 (1996)

14. P.B. Corkum, IEEE J. Quant. Electron., QE-21, 216 (1985)

15. I.V. Pogorelsky, I. Ben-Zvi, A. Van Steenbergen, R. Fernow, W.D. Kimura, and S.V. Bulanov, " $\mathrm{CO}_{2} \mathrm{Laser}$ Technology for Advanced Particle Accelerators", these Proceedings.

16. S.V. Bulanov, G.I. Dudnikova, N.M. Naumova, F. Pegoraro, I.V. Pogorelsky, V.A.Vshivkov, "Charged Particle Acceleration in Nonuniform Plasmas", these Proceedings. 